\title{
Microfluidic shear assays to distinguish between bacterial adhesion and attachment strength on stiffness-tunable silicone substrates
}

\section{Supplementary Information}

\section{Supporting calculations for pressure within microfluidic channels}

Pressure within the microchannel can be obtained by invoking the Bernoulli's equation with head loss, in which $p_{1}$ and $v_{1}$ represent the pressure and velocity at the inlet of the microchannel while $p_{2}$ and $v_{2}$ represent the pressure and velocity at the outlet. $\rho, g, f, L$, and $D_{H}$ represent fluid density, gravitational acceleration, friction factor, channel length, and hydrodynamic diameter, respectively:

$$
\frac{p_{1}}{\rho g}+\frac{v_{1}{ }^{2}}{2 g}=\frac{p_{2}}{\rho g}+\frac{v_{2}{ }^{2}}{2 g}+f \frac{L v^{2}}{D_{H} 2 g}
$$

For a wide rectangular channel, the hydrodynamic diameter can be approximated by the following standard equation, in which $a$ and $b$ are the sides of the channel:

$$
D_{H}=\frac{2 a b}{a+b}
$$

Thus, the microchannel in this study, which has width of $600 \mu \mathrm{m}$ and height of $100 \mu \mathrm{m}$, has a hydrodynamic diameter of $1.71 \times 10^{-4} \mathrm{~m}$. Friction factor in laminar flow (as is the case in this study) can be obtained from the following equation, where $R e$ is the Reynolds number and $\mu$ is the dynamic viscosity of the fluid:

$$
f=\frac{64}{R e}=\frac{64 \mu}{\rho v D_{H}}
$$

Since the channel dimensions do not change and the microchannel outlet is open to the atmosphere, $v_{1}$ $=v_{2}=v$ and $p_{1}-p_{2}=p_{\text {gauge }}$. The Bernoulli equation can then be rearranged to the form:

$$
p_{\text {gauge }}=\rho g f \frac{L v^{2}}{D_{H} 2 g}=\frac{32 \mu L v}{D_{H}{ }^{2}}
$$

In this study, the highest flowrate through the microchannel was $0.1 \mathrm{~mL} / \mathrm{min}$, which can be converted to a velocity of $v=0.028 \mathrm{~m} / \mathrm{s}$. Thus, $p_{\text {gauge }}$ was found to be $1084 \mathrm{~Pa}$ for the highest flowrates tested of $0.1 \mathrm{~mL} / \mathrm{min}$. This gauge pressure matches well with experimental results reported in literature for similar flowrates and channel geometries ${ }^{1-2}$.

Our simulations found deformation of the side walls of the microchannel to be several orders of magnitude smaller than any vertical deformation so side deformations are not reported. The total vertical deformation at the centre of the microchannel is shown in Figure $3 \mathrm{C}$ in response to various gauge pressures in the microchannel. For instance, pressure of $1084 \mathrm{~Pa}$ (corresponding to the high shear flowrate of $0.1 \mathrm{~mL} / \mathrm{min}$, at the fluid channel entrance) causes a total vertical deformation of $\sim 8 \mu \mathrm{m}$ when the stiffness-tunable layer of PDMS was soft, and $\sim 1.5 \mu \mathrm{m}$ when the stiffness-tunable PDMS was stiff. In comparison, the medium shear flowrate of $0.05 \mathrm{~mL} / \mathrm{min}$ produces $541 \mathrm{~Pa}$, which would vertically deform the microchannel by $\sim 4 \mu \mathrm{m}$ and $\sim 0.7 \mu \mathrm{m}$, respectively for a stiff PDMS and soft PDMS stiffnesstunable layer. 


\section{Supplementary References}

1. Hardy, B. S.; Uechi, K.; Zhen, J.; Pirouz Kavehpour, H., The deformation of flexible PDMS microchannels under a pressure driven flow. Lab on a Chip 2009, 9 (7), 935-938.

2. Gervais, T.; El-Ali, J.; Günther, A.; Jensen, K. F., Flow-induced deformation of shallow microfluidic channels. Lab on a Chip 2006, 6 (4), 500-507. 